\title{
A CONSTRUCTION OF LATTICES IN SPLITTABLE SOLVABLE LIE GROUPS
}

\author{
TAKUMI YAMADA
}

\begin{abstract}
In this paper, we consider a unified constructions of lattices in splittable solvable Lie groups.
\end{abstract}

\section{Introduction}

Let $G$ be a connected solvable Lie group. A discrete co-compact subgroup of $G$ is called a lattice in $G$. Auslander [2] has proven that a compact solvmanifold has a solvmanifold of the form $G / \Gamma$ as a finite covering, where $G$ is a simply connected solvable Lie group, and $\Gamma$ is a lattice in $G$. It is well known that a nilpotent Lie group has a lattice if and only if its Lie algebra has a basis with respect to which the constants of structure are rational. Moreover, the de Rham cohomology groups of a compact nilmanifold $N / \Gamma$ are isomorphic to the cohomology groups of the Lie algebra $n$ of $N$ ([7]). In particular, the de Rham cohomology groups of a compact nilmanifold are independent of lattices.

In the case of non-nilpotent solvable Lie groups, it is not easy to check the existence of a lattice. The de Rham cohomology groups of a compact solvmanifold $G / \Gamma$ are not isomorphic to the cohomology groups of the Lie algebra $g$ of $G$ in general. Two solvmanifolds $G_{1} / \Gamma_{1}$ and $G_{2} / \Gamma_{2}$ with isomorphic fundamental groups are diffeomorphic (see [8, Theorem 3.6]). Auslander also have proven that a Wang group is pre-divible, then it is isomorphic to a lattice in some simply connected solvable Lie group (see [2] for details). On the other hand, it is also important to construct a lattice in a given simply connected solvable Lie group (see e.g., [4, Examples 2, 3]). In the papers [11], [9], Sawai and the author have constructed lattices in splittable solvable Lie groups. However, the constructions in [11], [9] seem somewhat technical.

In this paper, we consider a unified construction of lattices in splittable solvable Lie groups by using the following theorem.

2010 Mathematics Subject Classification. Primary 22E25; Secondary 22E40.

Key words and phrases. Solvable Lie group, lattice, homogeneous space.

Received May 27, 2015; revised October 7, 2015. 
MAIN TheOREM. Let $G=N \rtimes_{\varphi} \mathbf{R}^{s}$ be a simply connected splittable solvable Lie group, where $N$ is the nilradical of $G$. Then $G$ has a splittable lattice $\Gamma=$ $\Gamma_{N} \rtimes_{\varphi} L$, where $\Gamma_{N}$ is a lattice in $N$ and $L$ is a lattices in $\mathbf{R}^{s}$, if and only if there exists a Q-algebra $\mathrm{n}_{0}$ of $\mathrm{n}$, and a lattice $L$ in $\mathbf{R}^{s}$ such that $d \varphi(L) \subset \operatorname{Aut}\left(\mathrm{n}_{0}\right)$ and $d \varphi(t)(t \in L)$ acts as an integer unimodular matrix with respect to a basis of $\mathrm{n}$ contained in $\mathrm{n}_{0}$.

The theorem can be considered as a weak version of Auslander's result [2, pp. 248-pp. 249]. However, it seems that a complete proof has not been published.

\section{Necessary and sufficient conditions for the existence of splittable lattices}

In this section, we consider a necessary and sufficient condition for the existence of splittable lattices in a splittable solvable Lie group.

There exists a necessary and sufficient condition of the existence for a lattice in a given nilpotent Lie group ([8, Theorem 2.12.]).

THEOREM $2.1([8])$. Let $N$ be a simply connected nilpotent Lie group, and $\mathrm{n}$ its Lie algebra. Suppose that $\mathrm{n}$ has a basis with respect to which the constants of structure are rational. Let $\mathrm{n}_{0}$ be the vector space over $\mathbf{Q}$ spanned by this basis; if $\mathscr{L}$ is any lattice of maximal rank in $\mathrm{n}$ contained in $\mathrm{n}_{0}$, and $\exp : \mathfrak{n} \rightarrow N$ is the exponential map, then the group generated by $\exp \mathscr{L}$ is a lattice in $N$. Conversely, if $\Gamma_{N}$ is a lattice in $N$, then the $\mathbf{Z}$-span of $\exp ^{-1} \Gamma_{N}$ is a lattice $\mathscr{L}$ in the vector space $\mathrm{n}$ such that the structural constants of $\mathrm{n}$ with respect to any basis contained in $\mathscr{L}$ belong to $\mathbf{Q}$.

Let $\Gamma$ be a lattice in a connected solvable Lie group $G$, and $N$ the nilradical of $G$. Then, the following theorem is well-known.

Theorem 2.2 (Mostow [6]). $N \cap \Gamma$ is a lattice in $N$.

Let $G$ be a simply connected solvable Lie group, and $N$ the nilradical of $G$. Then $G$ satisfies the exact sequence

$$
1 \rightarrow N \rightarrow G \rightarrow \mathbf{R}^{s} \rightarrow 1 .
$$

We say that $G$ is splittable if the short exact sequence splits. It is well-known that if $G$ is splittable, then $G$ is isomorphic to a semi-direct product $N \rtimes_{\varphi} \mathbf{R}^{s}$, where $\varphi: \mathbf{R}^{s} \rightarrow \operatorname{Aut}(N)$ is a homomorphism.

Then, we have the following corollary.

COROllary 2.3. Let $G=N \rtimes_{\varphi} \mathbf{R}^{s}$ be a simply connected splittable solvable Lie group, where $N$ the nilradical of $G$, and $\Gamma=\Gamma_{N} \rtimes_{\varphi} L$ its splittable lattice. 
Then $C^{k}(N) \rtimes_{\varphi} \mathbf{R}^{s}$ has a lattice, where $\left\{C^{k}(N)\right\}$ the descending central series for $N$.

Proof. $\left(C^{k}(N) \cap \Gamma_{N}\right) \rtimes_{\varphi} L$ is a lattice in $C^{k}(N) \rtimes_{\varphi} \mathbf{R}^{s}$.

THEOREM 2.4. Let $G=N \rtimes_{\varphi} \mathbf{R}^{s}$ be a simply connected splittable solvable Lie group, where $N$ is the nilradical of $G$. Then $G$ has a splittable lattice $\Gamma=$ $\Gamma_{N} \rtimes_{\varphi} L$, where $\Gamma_{N}$ is a lattice in $N$ and $L$ is a lattices in $\mathbf{R}^{s}$, if and only if there exists a Q-algebra $\mathrm{n}_{0}$ of $\mathrm{n}$, and a lattice $L$ in $\mathbf{R}^{s}$ such that $d \varphi(L) \subset$ Aut $\left(\mathrm{n}_{0}\right)$ and $d \varphi(t)(t \in L)$ acts as an integer unimodular matrix with respect to a basis of $\mathrm{n}$ contained in $\mathrm{n}_{0}$.

Proof. Assume first that there exists a $\mathbf{Q}$-algebra $\mathrm{n}_{0}$ of $\mathrm{n}$, and lattice $L$ in $\mathbf{R}^{s}$ such that $d \varphi(L) \subset \operatorname{Aut}\left(\mathrm{n}_{0}\right)$ and $d \varphi(t)(t \in L)$ acts as an integer unimodular matrices with respect to a basis of $\mathrm{n}$ contained in $\mathfrak{n}_{0}$. Let $\mathscr{L} \subset \mathfrak{n}_{0}$ be a lattice of maximal rank in $\mathrm{n}$. By Theorem 2.1, the group $\Gamma_{N}$ generated by $\exp \mathscr{L}$ is a lattice in $N$. Let $X \in \mathscr{L}$. Then $d \varphi(t)(X) \in \mathscr{L}$ for $t \in L$. Thus $\exp (d \varphi(t) X)=\varphi(t)(\exp X) \in \Gamma_{N}$. Since $\quad(n, t) \cdot\left(n^{\prime}, t^{\prime}\right)=\left(n \varphi(t)\left(n^{\prime}\right), t t^{\prime}\right), \quad$ and $(n, t)^{-1}=\left(\varphi\left(t^{-1}\right)\left(n^{-1}\right), t^{-1}\right)$ for $(n, t),\left(n^{\prime}, t^{\prime}\right) \in N \rtimes_{\varphi} \mathbf{R}^{s}, \Gamma=\Gamma_{N} \rtimes_{\varphi} L$ is a discrete subgroup of $G=N \rtimes_{\varphi} \mathbf{R}^{s}$. Since a fiber bundle

$$
N / N \cap \Gamma=N / \Gamma_{N} \rightarrow G / \Gamma \rightarrow G / N \Gamma=(G / N) /(N \Gamma / N)
$$

has a compact base and a compact fiber, $G / \Gamma$ is compact (note that $N \Gamma$ is closed by Theorem 1.13 in [8]). Thus, $\Gamma$ is a lattice in $G$.

Conversely, assume that $\Gamma=\Gamma_{N} \rtimes_{\varphi} L$ is a lattice in $G$. Put

$$
\mathscr{L}=\operatorname{span}_{\mathbf{Z}}\left\{X \in \mathfrak{n} \mid X \in \exp ^{-1} \Gamma_{N}\right\} .
$$

Then

$$
\mathscr{L}=\operatorname{span}_{\mathbf{Z}}\left\{X_{1}, \ldots, X_{n}\right\} \subset \mathfrak{n},
$$

where $n=\operatorname{dim} n$ by Theorem 2.1. Moreover, $\mathscr{L}$ is a lattice in the vector space $n$ such that the structural constants of $n$ with respect to any basis contained in $\mathscr{L}$ belong to $\mathbf{Q}$ by Theorem 2.1. Since $\Gamma_{N} \rtimes_{\varphi} L$ is a subgroup, we see that for every $t \in L$ and $i=1, \ldots, n,(e, t) \cdot\left(\exp X_{i}, 0\right)=\left(\varphi(t) \exp X_{i}, t\right) \in \Gamma_{N} \rtimes_{\varphi} L . \quad$ Thus,

$$
d \varphi(t) X_{i} \in \mathscr{L}=\operatorname{span}_{\mathbf{Z}}\left\{X_{1}, \ldots, X_{n}\right\} .
$$

Thus, $L$ acts as integer unimodular matrices with respect to $X_{1}, \ldots, X_{n}$.

The following lemma is obvious, however, it is useful to construct lattices (see Section 3).

Lemma 2.5. Let $G=N \rtimes_{\varphi} \mathbf{R}^{s}$ be a simply connected splittable solvable Lie group, where $N$ is the nilradical of $G$. We assume that

(1) $N$ is $(r+1)$-step and there exists a Q-algebra $\mathfrak{n}_{0}$ of $\mathrm{n}$. 
(2) There exists a lattice $L=\mathbf{Z t}_{1}+\cdots+\mathbf{Z t}_{s}$ in $\mathbf{R}^{s}$ such that $d \varphi(L) \subset \operatorname{Aut}\left(\mathfrak{n}_{0}\right)$ and $d \varphi(t) \quad(t \in L)$ acts as an integer unimodular matrix with respect to a basis of $\mathrm{n}$ contained in $\mathrm{n}_{0}$.

(3) $d \varphi\left(\mathbf{t}_{i}\right)(i=1, \ldots, s)$ are semi-simple.

Then there exist vector spaces $\mathfrak{m}_{k}(k=0, \ldots, r)$ which satisfy the following conditions:

(1) $C^{k-1}(\mathfrak{n})=\mathfrak{m}_{k-1} \oplus C^{k}(\mathfrak{n})$ for $k=1, \ldots, r+1$.

(2) $d \varphi\left(\mathbf{t}_{i}\right)\left(\mathfrak{m}_{k}\right) \subset \mathfrak{m}_{k}$ for $i=1, \ldots, s$.

(3) $d \varphi\left(\mathbf{t}_{i}\right): \mathfrak{m}_{k} \rightarrow \mathfrak{m}_{k}$ is unimodular for $i=1, \ldots, s$.

Proof. We only prove the case of $s=1$, because $d \varphi\left(\mathbf{t}_{1}\right), \ldots, d \varphi\left(\mathbf{t}_{s}\right)$ are simultaneously diagonalizable. We write $L=t_{0} \mathbf{Z}$. Let $\mathfrak{m}_{r}=C^{r}(\mathfrak{n})$. Then, it is obvious that $\mathrm{m}_{r}$ satisfies the conditions (2), (3) by Corollary 2.3. Assume that there exist $\mathfrak{m}_{k}, \ldots, \mathfrak{m}_{r}$ which satisfy the conditions (2), (3). Since $d \varphi\left(t_{0}\right)$ is semisimple, and $d \varphi\left(t_{0}\right)\left(C^{k-1}(\mathrm{n})\right) \subset C^{k-1}(\mathfrak{n})$, there exist

$$
X_{1}^{(k-1)}, \ldots, X_{i_{k-1}}^{(k-1)} \in C^{k-1}(\mathfrak{n}) \backslash C^{k}(\mathfrak{n})
$$

such that

$$
C^{k-1}(\mathrm{n})=\operatorname{span}\left\{X_{1}^{(k-1)}, \ldots, X_{i_{k-1}}^{(k-1)}\right\} \oplus C^{k}(\mathfrak{n}), \quad d \varphi\left(t_{0}\right) X_{j}^{(k-1)}=\lambda_{j}^{(k-1)} X_{j}^{(k-1)}
$$

for $j=1, \ldots, i_{k-1}$, where $\lambda_{j}^{(k-1)} \in \mathbf{R}$. Put

$$
\mathfrak{m}_{k-1}=\operatorname{span}\left\{X_{1}^{(k-1)}, \ldots, X_{i_{k-1}}^{(k-1)}\right\} .
$$

Then, $d \varphi\left(t_{0}\right)\left(\mathfrak{m}_{k-1}\right) \subset \mathfrak{m}_{k-1}$. Since $\left.d \varphi(t)\right|_{C^{k-1}(\mathfrak{n})}$ and $\left.d \varphi(t)\right|_{C^{k}(\mathfrak{n})}$ are unimodular, $\left.d \varphi(t)\right|_{\mathfrak{m}_{k-1}}$ is also unimodular.

\section{Examples}

In this section, we construct lattices in splittable solvable Lie groups, which are famous, by using Theorem 2.4. Similarly as in this section, we can construct lattices in other solvable Lie groups.

Example 3.1 ([10], Inoue surface of type $S^{0}$ ). Let $\mathfrak{g}$ be a solvable Lie algebra given by

$$
\mathfrak{g}=\operatorname{span}\{T, X, Y, \bar{Y}\}
$$

with nontrivial structure equations

$$
[T, X]=a X, \quad[T, Y]=b Y, \quad[T, \bar{Y}]=\bar{b} \bar{Y},
$$

where $b \in \mathbf{C}$, and $e^{a}, e^{b}, e^{\bar{b}}$ are eigenvalues of $B \in S L(3, \mathbf{Z})$.

Let $\mathfrak{n}=\operatorname{span}\{X, Y, \bar{Y}\}$. Let $G$ be the simply connected solvable Lie group corresponding to $\mathfrak{g}$, and $N \subset G$ the simply connected nilpotent Lie group corresponding to $\mathrm{n}$. of $e^{b}$. Let

Let ${ }^{t}\left(a_{1}, a_{2}, a_{3}\right)$ be a real eigenvector of $e^{a}$, and ${ }^{t}\left(b_{1}, b_{2}, b_{3}\right)$ an eigenvector 


$$
P=\left(\begin{array}{lll}
a_{1} & a_{2} & a_{3} \\
b_{1} & b_{2} & b_{3} \\
\bar{b}_{1} & \bar{b}_{2} & \bar{b}_{3}
\end{array}\right) .
$$

Put $(\tilde{X}, \tilde{Y}, \tilde{Z})=(X, Y, \bar{Y}) P$. Then $B \in S L(3, \mathbf{Z})$ is the matrix representation of $\left.\exp a d(T)\right|_{\mathfrak{n}}$ with respect to $\tilde{X}, \tilde{Y}, \tilde{Z}$. Let $\mathscr{L}=\operatorname{span}_{\mathbf{Z}}\{\tilde{X}, \tilde{Y}, \tilde{Z}\}$, and $\Gamma_{N}$ the group generated by $\mathscr{L}$. By Theorem 2.4, $G$ has a lattice $\Gamma=\Gamma_{N} \rtimes \mathbf{Z}$.

Example 3.2 ([1], [9], Inoue surface of type $S^{+}$). Let $\mathfrak{g}_{1}$ be a solvable Lie algebra given by

$$
\mathfrak{g}_{1}=\operatorname{span}\{T, X, Y, Z\}
$$

with nontrivial structure equations

$$
[T, X]=X, \quad[T, Y]=-Y, \quad[X, Y]=Z .
$$

Let $\mathrm{n}=\operatorname{span}\{X, Y, Z\}$. Let $G_{1}$ be the simply connected solvable Lie group corresponding to $\mathfrak{g}_{1}$, and $N \subset G_{1}$ the simply connected nilpotent Lie group corresponding to $\mathrm{t}$.

Let $M \in S L(2, \mathbf{Z})$ be a unimodular matrix given by

$$
M=\left(\begin{array}{cc}
0 & -1 \\
1 & n
\end{array}\right) \in S L(2, \mathbf{Z}) .
$$

Then the characteristic polynomial of $M$ is $f(x)=x^{2}-n x+1$. Let $\lambda, \lambda^{-1}$ be the characteristic roots. Take $t_{0}=\log \lambda$, i.e., $e^{t_{0}}=\lambda$. Let $P=\left(\begin{array}{cc}1 & \lambda \\ 1 & \lambda^{-1}\end{array}\right)$. Then $P M P^{-1}=\left(\begin{array}{cc}\lambda & 0 \\ 0 & \lambda^{-1}\end{array}\right)$.

Let $\mathrm{m}_{0}=\operatorname{span}\{X, Y\}$, and $\mathrm{m}_{1}=\operatorname{span}\{Z\}$ (cf. Lemma 2.5). Put $(\tilde{X}, \tilde{Y})=$ $(X, Y) P$. Then $M \in S L(2, \mathbf{Z})$ is the matrix representation of exp $\left.\operatorname{ad}\left(t_{0} T\right)\right|_{\mathrm{m}_{0}}$ with respect to $\tilde{X}, \tilde{Y}$, and

$$
[\tilde{X}, \tilde{Y}]=\left[X+Y, \lambda X+\lambda^{-1} Y\right]=\left(\lambda^{-1}-\lambda\right) Z=|P| Z .
$$

Put $\tilde{Z}=|P| Z$. Then $\tilde{X}, \tilde{Y}, \tilde{Z}$ is a basis of the nilradical $\mathrm{n}$ of $\mathfrak{g}$ with respect to which the constants of structure are rational, and the matrix representation of $\left.\exp a d\left(t_{0} T\right)\right|_{\mathrm{n}}$ is an unimodular integer matrix. Let $\mathscr{L}=\operatorname{span}_{\mathbf{Z}}\{\tilde{X}, \tilde{Y}, \tilde{Z}\}$, and $\Gamma_{N}$ the group generated by $\mathscr{L}$. By Theorem $2.4, G_{1}$ has a lattice $\Gamma=\Gamma_{N} \rtimes t_{0} \mathbf{Z}$.

Next, we express the above lattice in $G_{1}$ explicitly. The solvable Lie group $G_{1}$ can be written as

$$
G_{1}=\left\{\left(\begin{array}{ccccc}
1 & -\frac{1}{2} y e^{t} & \frac{1}{2} x e^{-t} & 0 & z \\
0 & e^{t} & 0 & 0 & x \\
0 & 0 & e^{-t} & 0 & y \\
0 & 0 & 0 & 1 & t \\
0 & 0 & 0 & 0 & 1
\end{array}\right) \mid t, x, y, z \in \mathbf{R}\right\} \cong N \ltimes \mathbf{R}^{1}
$$


Thus, the solvable Lie group $G_{1}$ is isomorphic to $\left(\mathbf{R}^{2} \times \mathbf{R}^{1} \times \mathbf{R}^{1}, *\right)$, where

$$
\begin{aligned}
& \left(\mathbf{x}_{1}, z_{1}, t_{1}\right) *\left(\mathbf{x}_{2}, z_{2}, t_{2}\right) \\
& \quad=\left(\mathbf{x}_{1}+\left(\begin{array}{cc}
e^{t_{1}} & 0 \\
0 & e^{-t_{1}}
\end{array}\right) \mathbf{x}_{2}, z_{1}+\frac{1}{2}{ }^{t} \mathbf{x}_{1}\left(\begin{array}{cc}
0 & 1 \\
-1 & 0
\end{array}\right)\left(\begin{array}{cc}
e^{t_{1}} & 0 \\
0 & e^{-t_{1}}
\end{array}\right) \mathbf{x}_{2}+z_{2}, t_{1}+t_{2}\right) .
\end{aligned}
$$

Then, a subgroup

$$
\Gamma=\left\{\left(P \mathbf{m}, \frac{1}{2}|P| n\right) \mid \mathbf{m} \in \mathbf{Z}^{2}, n \in \mathbf{Z}\right\} \rtimes t_{0} \mathbf{Z}
$$

is a lattice in $\left(\mathbf{R}^{2} \times \mathbf{R}^{1} \times \mathbf{R}^{1}, *\right) \cong G_{1}$. Indeed, note that

$$
\begin{aligned}
& \exp (a \tilde{X}+b \tilde{Y}+c \tilde{Z}) \exp \left(a^{\prime} \tilde{X}+b^{\prime} \tilde{Y}+c^{\prime} \tilde{Z}\right) \\
& \quad=\exp \left(\left(a+a^{\prime}\right) \tilde{X}+\left(b+b^{\prime}\right) \tilde{Y}+\left(c+\frac{1}{2} a b^{\prime}-\frac{1}{2} a^{\prime} b+c^{\prime}\right) \tilde{Z}\right),
\end{aligned}
$$

and this product coincides with the product of $N \subset G_{1}$. By the first canonical coordinates, $\exp (a \tilde{X}+b \tilde{Y}+c \tilde{Z}) \mapsto\left(\left(\begin{array}{l}a \\ b\end{array}\right), c\right)$, we have

$$
\Gamma_{N} \rightarrow\left\{\left(\mathbf{m}, \frac{1}{2} n\right) \mid \mathbf{m} \in \mathbf{Z}^{2}, n \in \mathbf{Z}\right\} .
$$

Since $(\tilde{X}, \tilde{Y})=(X, Y) P$, and $\tilde{Z}=|P| Z$, we see that $\Gamma$ is a lattice in $G_{1}$.

Example 3.3 ([4], [9]). Let $\mathfrak{g}_{2}$ be a solvable Lie algebra given by

$$
\mathfrak{g}_{2}=\operatorname{span}\left\{T, X_{1}, X_{2}, X_{3}, Z_{1}, Z_{2}, Z_{3}\right\}
$$

with nontrivial equations

$$
\begin{array}{lll}
{\left[X_{1}, X_{2}\right]=Z_{3},} & {\left[X_{2}, X_{3}\right]=Z_{1},} & {\left[X_{3}, X_{1}\right]=Z_{2},} \\
{\left[T, X_{1}\right]=-a_{1} X_{1},} & {\left[T, X_{2}\right]=-a_{2} X_{2},} & {\left[T, X_{3}\right]=-a_{3} X_{3},} \\
{\left[T, Z_{1}\right]=a_{1} Z_{1},} & {\left[T, Z_{2}\right]=a_{2} Z_{2},} & {\left[T, Z_{3}\right]=a_{3} Z_{3},}
\end{array}
$$

where $a_{1}, a_{2}, a_{3}$ are distinct real numbers such that $a_{1}+a_{2}+a_{3}=0$, and $t_{0} \in \mathbf{R}$ and $m, n \in \mathbf{N}$ satisfy that $e^{a_{1} t_{0}}, e^{a_{2} t_{0}}, e^{a_{3} t_{0}}$ are distinct roots of the polynomial $f(x)=x^{3}-m x^{2}+n x-1$ (cf. [9, Thereom 1]). Let $\mathrm{n}=\operatorname{span}\left\{X_{1}, X_{2}, X_{3}, Z_{1}, Z_{2}\right.$, $\left.Z_{3}\right\}$. Let $G_{2}$ be the simply connected solvable Lie group corresponding to $\mathfrak{g}_{2}$, and $N \subset G_{2}$ the simply connected nilpotent Lie group corresponding to $\mathrm{n}$.

Let $B$ be a unimodular matrix given by

$$
B=\left(\begin{array}{ccc}
0 & 0 & 1 \\
1 & 0 & -n \\
0 & 1 & m
\end{array}\right) .
$$


Then, the characteristic polynomial of $B$ is $f(x)=x^{3}-m x^{2}+n x-1$. Put $\lambda_{i}=e^{a_{i} t_{0}}(i=1,2,3)$, and $P=\left(\begin{array}{ccc}1 & \lambda_{1} & \lambda_{1}^{2} \\ 1 & \lambda_{2} & \lambda_{2}^{2} \\ 1 & \lambda_{3} & \lambda_{3}^{2}\end{array}\right)$. Then $P B P^{-1}=\left(\begin{array}{ccc}\lambda_{1} & 0 & 0 \\ 0 & \lambda_{2} & 0 \\ 0 & 0 & \lambda_{3}\end{array}\right)$. Let $\mathrm{m}_{0}=\operatorname{span}\left\{X_{1}, X_{2}, X_{3}\right\}$, and $\mathrm{m}_{1}=\operatorname{span}\left\{Z_{1}, Z_{2}, Z_{3}\right\}$. Put

$$
\left(\tilde{X}_{1}, \tilde{X}_{2}, \tilde{X}_{3}\right)=\left(X_{1}, X_{2}, X_{3}\right) P \text {. }
$$

Then $B^{-1} \in S L(3, \mathbf{Z})$ is the matrix representation of $\left.\exp a d\left(t_{0} T\right)\right|_{\mathfrak{m}_{0}}$ with respect to $\tilde{X}_{1}, \tilde{X}_{1}, \tilde{X}_{3}$. Moreover, let

$$
\tilde{Z}_{3}=\left[\tilde{X}_{1}, \tilde{X}_{2}\right], \quad \tilde{Z}_{1}=\left[\tilde{X}_{2}, \tilde{X}_{3}\right], \quad \tilde{Z}_{2}=\left[\tilde{X}_{3}, \tilde{X}_{1}\right] .
$$

Then we can easily see that

$$
\left(\tilde{Z}_{1}, \tilde{Z}_{2}, \tilde{Z}_{3}\right)=\left(Z_{1}, Z_{2}, Z_{3}\right)|P|^{t} P^{-1},
$$

and ${ }^{t} B$ is the matrix representation of $\left.\exp \operatorname{ad}\left(t_{0} T\right)\right|_{\mathrm{m}_{1}}$ with respect to $\tilde{Z}_{1}, \tilde{Z}_{2}$, $\tilde{Z}_{3}$. Then $\tilde{X}_{1}, \tilde{X}_{1}, \tilde{X}_{3}, \tilde{Z}_{1}, \tilde{Z}_{1}, \tilde{Z}_{2}, \tilde{Z}_{3}$ is a basis of the nilradical $\mathfrak{n}$ of $\mathfrak{g}$ with respect to which the constants of structure are rational, and the matrix representation of $\left.\exp a d\left(t_{0} T\right)\right|_{\mathfrak{n}}$ is an unimodular integer matrix. Let $\mathscr{L}=\operatorname{span}_{\mathbf{Z}}\left\{\tilde{X}_{1}, \tilde{X}_{2}\right.$, $\left.\tilde{X}_{3}, \tilde{Z}_{1}, \tilde{Z}_{2}, \tilde{Z}_{3}\right\}$, and $\Gamma_{N}$ the group generated by $\mathscr{L}$. By Theorem $2.4, G_{2}$ has a lattice $\Gamma=\Gamma_{N} \rtimes t_{0} \mathbf{Z}$.

Next, we express the above lattice in $G_{2}$ explicitly. The solvable Lie group $G_{2}$ corresponding to $\mathfrak{g}_{2}$ can be written as

$$
\begin{aligned}
G_{2} & =\left\{\left(\begin{array}{cccccccc}
e^{a_{1} t} & 0 & 0 & 0 & -\frac{1}{2} x_{3} e^{-a_{2} t} & \frac{1}{2} x_{2} e^{-a_{3} t} & 0 & z_{1} \\
0 & e^{a_{2} t} & 0 & \frac{1}{2} x_{3} e^{-a_{1} t} & 0 & -\frac{1}{2} x_{1} e^{-a_{3} t} & 0 & z_{2} \\
0 & 0 & e^{a_{3} t} & -\frac{1}{2} x_{2} e^{-a_{1} t} & \frac{1}{2} x_{1} e^{-a_{2} t} & 0 & 0 & z_{3} \\
0 & 0 & 0 & e^{-a_{1} t} & 0 & 0 & 0 & x_{1} \\
0 & 0 & 0 & 0 & e^{-a_{2} t} & 0 & 0 & x_{2} \\
0 & 0 & 0 & 0 & 0 & e^{-a_{3} t} & 0 & x_{3} \\
0 & 0 & 0 & 0 & 0 & 0 & 1 & t \\
0 & 0 & 0 & 0 & 0 & 0 & 0 & 1
\end{array}\right) \mid t, x_{i}, z_{i} \in \mathbf{R}\right\} \\
& \cong N \ltimes \mathbf{R} .
\end{aligned}
$$

Thus, the solvable Lie group $G_{2}$ is isomorphic to $\left(\mathbf{R}^{3} \times \mathbf{R}^{3} \times \mathbf{R}^{1}, *\right)$, where

$$
\begin{aligned}
& \left(\mathbf{x}_{1}, \mathbf{z}_{1}, t_{1}\right) *\left(\mathbf{x}_{2}, \mathbf{z}_{2}, t_{2}\right) \\
& =\left(\mathbf{x}_{1}+\left(\begin{array}{ccc}
e^{-a_{1} t_{1}} & 0 & 0 \\
0 & e^{-a_{2} t_{1}} & 0 \\
0 & 0 & e^{-a_{3} t_{1}}
\end{array}\right) \mathbf{x}_{2}, \mathbf{z}_{1}+A\left(\mathbf{x}_{1}\right)\left(\begin{array}{ccc}
e^{-a_{1} t_{1}} & 0 & 0 \\
0 & e^{-a_{2} t_{1}} & 0 \\
0 & 0 & e^{-a_{3} t_{1}}
\end{array}\right) \mathbf{x}_{2}\right. \\
& \left.+\left(\begin{array}{ccc}
e^{a_{1} t_{1}} & 0 & 0 \\
0 & e^{a_{2} t_{1}} & 0 \\
0 & 0 & e^{a_{3} t_{1}}
\end{array}\right) \mathbf{z}_{2}, t_{1}+t_{2}\right)
\end{aligned}
$$


where $A(\mathbf{x})=\frac{1}{2}\left(\begin{array}{ccc}0 & -x_{3} & x_{2} \\ x_{3} & 0 & -x_{1} \\ -x_{2} & x_{1} & 0\end{array}\right)$ for $\mathbf{x}={ }^{t}\left(x_{1}, x_{2}, x_{3}\right)$. Then, a subgroup

is a lattice in $G_{2}$.

$$
\Gamma=\left\{\left(P \mathbf{m}, \frac{1}{2}|P|^{t} P^{-1} \mathbf{n}\right) \mid \mathbf{m}, \mathbf{n} \in \mathbf{Z}^{3}\right\} \rtimes t_{0} \mathbf{Z}
$$

Remark 3.4. The assumptions with respect to $M, P$, and $B$ are not essential. Indeed, let $M \in S L(2, \mathbf{Z})$ be a unimodular matrix with distinct real positive eigenvalues, say, $\lambda, 1 / \lambda$. Take $t_{0}=\log \lambda$. Let $P \in G L(2, \mathbf{R})$ be a matrix which satisfies

$$
P M P^{-1}=\left(\begin{array}{cc}
e^{t_{0}} & 0 \\
0 & e^{-t_{0}}
\end{array}\right)
$$

Then,

$$
\Gamma=\left\{\left(P \mathbf{m}, \frac{1}{2}|P| n\right) \mid \mathbf{m} \in \mathbf{Z}^{2}, n \in \mathbf{Z}\right\} \rtimes t_{0} \mathbf{Z}
$$

is a lattice in $G_{1}$. Similarly, we have the same argument in Example 3.3.

Remark 3.5. We can explain the isomorphisms of Lie groups in $[9$, pp. 3127, pp. 3132]. Let $V^{n}$ be an $n$ dimensional real vector space, and $B: V \rightarrow \operatorname{Hom}_{\mathbf{R}}\left(V, \mathbf{R}^{m}\right)$ a linear mapping. We define a multiplication on the set $N(B)=V \times \mathbf{R}^{m}$ by

$$
\left(v_{1}, z_{1}\right) *\left(v_{2}, z_{2}\right)=\left(v_{1}+v_{2}, z_{1}+z_{2}+\left(B\left(v_{1}\right)\right)\left(v_{2}\right)\right), \quad v_{i} \in V, z_{i} \in \mathbf{R}^{m} \quad(i=1,2) .
$$

It is straightforward to verify that $(v, z)^{-1}=(-v,-z+(B(v))(v))$. Since $Z=$ $\{0\} \times \mathbf{R}^{m}$ is a normal subgroup and $N(B) / Z \cong V$ is abelian, $N(B)$ is a 2-step nilpotent Lie group. If $v={ }^{t}\left(x_{1}, \ldots, x_{n}\right)=\mathbf{x}$ and $B(\mathbf{x})=\left(a_{i j}(\mathbf{x})\right), i, j=1, \ldots, n$ relative to a basis of $V$, then

$$
(\mathbf{x}, z) \mapsto\left(\begin{array}{ccc}
I_{m} & B(\mathbf{x}) & z \\
0 & I_{n} & \mathbf{x} \\
0 & 0 & 1
\end{array}\right)
$$

where $I_{n}$ is the $n \times n$ unit matrix, is a faithful representation of $N(B)$. Let $m=n$. Then we can write $B(\mathbf{x})=A(\mathbf{x})+S(\mathbf{x})$, where $A(\mathbf{x})$ is the alternate matrix and $S(\mathbf{x})$ is the symmetric matrix corresponding to $B(\mathbf{x})$, respectively. Note that we can consider a 2 -step nilpotent Lie group $N(A)$. Let a subscript 0 denote that the element is in $N(A)$ and a subscript 1 denote that the element is in $N(B)=N(A+S)$. Let

$$
\pi_{S}\left((\mathbf{x}, z)_{0}\right)=\left(\mathbf{x}, z+\frac{1}{2}(S(\mathbf{x}))(\mathbf{x})\right)_{1} .
$$

If $\left(S\left(\mathbf{x}_{1}\right)\right)\left(\mathbf{x}_{2}\right)=\left(S\left(\mathbf{x}_{2}\right)\right)\left(\mathbf{x}_{1}\right)$ for each $\mathbf{x}_{1}, \mathbf{x}_{2} \in \mathbf{R}^{n}$, then $\pi_{S}$ is an isomorphism. 
In the case of $m=1, B$ can be considered as a bilinear form. Hence, we write $\left(B\left(v_{1}\right)\right)\left(v_{2}\right)=B\left(v_{1}, v_{2}\right)$. If $v={ }^{t}\left(x_{1}, \ldots, x_{n}\right)=\mathbf{x} \quad$ and $B=\left(a_{i j}\right), \quad i, j=$ $1, \ldots, n$ relative to a basis of $V$, then we can write the above representation as

$$
(\mathbf{x}, z) \mapsto\left(\begin{array}{ccc}
1 & { }^{t} \mathbf{x} B & z \\
0 & I_{n} & \mathbf{x} \\
0 & 0 & 1
\end{array}\right)
$$

Let $B=A+S$, where $A$ is the alternating bilinear form and $S$ is the symmetric bilinear form corresponding to $B(\mathbf{x})$, respectively. Then we see that $\pi_{S}$ is always an isomorphism (see [3, pp. 1-pp. 2] for $m=n=1$ ).

For example, let $G$ be the following solvable Lie group.

$$
G=N(B) \rtimes \mathbf{R}=\left\{\left(\begin{array}{ccccc}
1 & 0 & x e^{-t} & 0 & z \\
0 & e^{t} & 0 & 0 & x \\
0 & 0 & e^{-t} & 0 & y \\
0 & 0 & 0 & 1 & t \\
0 & 0 & 0 & 0 & 1
\end{array}\right) \mid t, x, y, z \in \mathbf{R}\right\},
$$

where

$$
N(B)=\left\{\left(\begin{array}{cccc}
1 & 0 & x & z \\
0 & 1 & 0 & x \\
0 & 0 & 1 & y \\
0 & 0 & 0 & 1
\end{array}\right) \mid x, y, z \in \mathbf{R}\right\}
$$

Hence, $B=\left(\begin{array}{ll}0 & 1 \\ 0 & 0\end{array}\right)$. Then, the alternate part $A$ of $B$ is $\frac{1}{2}\left(\begin{array}{cc}0 & 1 \\ -1 & 0\end{array}\right)$. Thus,
we have

$$
G \cong N(A) \rtimes \mathbf{R}=\left\{\left(\begin{array}{ccccc}
1 & -\frac{1}{2} y e^{t} & \frac{1}{2} x e^{-t} & 0 & z \\
0 & e^{t} & 0 & 0 & x \\
0 & 0 & e^{-t} & 0 & y \\
0 & 0 & 0 & 1 & t \\
0 & 0 & 0 & 0 & 1
\end{array}\right) \mid t, x, y, z \in \mathbf{R}\right\}
$$

because $\exp a d(t T) \circ \pi_{S}=\pi_{S} \circ \exp a d(t T)$ for any $t \in \mathbf{R}$.

Similarly, let $B(\mathbf{x})=\left(\begin{array}{ccc}0 & 0 & x_{2} \\ x_{3} & 0 & 0 \\ 0 & x_{1} & 0\end{array}\right)$. Then, $A(\mathbf{x})=\frac{1}{2}\left(\begin{array}{ccc}0 & -x_{3} & x_{2} \\ x_{3} & 0 & -x_{1} \\ -x_{2} & x_{1} & 0\end{array}\right)$. Thus, we have an isomorphism in [9, pp. 3127].

\section{Lattice of a family of solvable Lie groups}

Let $B_{1}, \ldots, B_{n-1} \in S L(n, \mathbf{Z})$ be matrices which satisfy that $B_{i} B_{j}=B_{j} B_{i}$ for each $i, j$, and each eigenvalue is positive. Let $P \in G L(n, \mathbf{R})$ be a matrix 
such that

$$
P B_{i} P^{-1}=\left(\begin{array}{ccc}
\alpha_{1}^{i} & & 0 \\
& \ddots & \\
0 & & \alpha_{n}^{i}
\end{array}\right)=\left(\begin{array}{ccc}
e^{\log \alpha_{1}^{i}} & & 0 \\
& \ddots & \\
0 & & e^{\log \alpha_{n}^{i}}
\end{array}\right)
$$

for each $i$ (note that $\sum_{k=1}^{n} \log \alpha_{k}^{i}=0$ ). If $\left(\begin{array}{c}\log \alpha_{1}^{i} \\ \vdots \\ \log \alpha_{n-1}^{i}\end{array}\right) \in \mathbf{R}^{n-1}(i=1, \ldots, n-1)$ are linearly independent, then a solvable Lie group

$$
G=\left\{\left(\begin{array}{cccccc}
e^{t_{1}} & 0 & \cdots & 0 & 0 & x_{1} \\
0 & \ddots & & \vdots & 0 & \vdots \\
\vdots & & e^{t_{n-1}} & 0 & \vdots & \vdots \\
0 & \cdots & 0 & e^{-\left(t_{1}+\cdots+t_{n-1}\right)} & 0 & x_{n} \\
0 & \cdots & \cdots & 0 & I_{n-1} & \mathbf{t} \\
0 & \cdots & \cdots & 0 & 0 & 1
\end{array}\right) \mid \mathbf{t} \in \mathbf{R}^{n-1}, x_{1}, \ldots, x_{n} \in \mathbf{R}\right\}
$$

where $\mathbf{t}={ }^{t}\left(t_{1}, \ldots, t_{n-1}\right)$, has a lattice. Indeed, note that $G \cong \mathbf{R}^{n} \rtimes_{\varphi} \mathbf{R}^{n-1}$, where $\varphi\left(t_{1}, \ldots, t_{n-1}\right)=\operatorname{diag}\left(e^{t_{1}}, \ldots, e^{t_{n-1}}, e^{-\left(t_{1}+\cdots+t_{n-1}\right)}\right)$. Then,

$$
\Gamma=\left\{P\left(\begin{array}{c}
m_{1} \\
\vdots \\
m_{n}
\end{array}\right) \mid m_{i} \in \mathbf{Z}\right\} \rtimes_{\varphi}\left(\mathbf{Z}\left(\begin{array}{c}
\log \alpha_{1}^{1} \\
\vdots \\
\log \alpha_{n-1}^{1}
\end{array}\right) \times \cdots \times \mathbf{Z}\left(\begin{array}{c}
\log \alpha_{1}^{n-1} \\
\vdots \\
\log \alpha_{n-1}^{n-1}
\end{array}\right)\right)
$$

is a lattice in $G \cong \mathbf{R}^{n} \rtimes_{\varphi} \mathbf{R}^{n-1}$ by Thereom 2.4 .

Remark 4.1. The solvable Lie group has a left invariant contact form. a lattice.

In the case of $n=3$, if $\frac{\log \alpha_{1}^{1}}{\log \alpha_{2}^{1}} \neq \frac{\log \alpha_{1}^{2}}{\log \alpha_{2}^{2}}$, then the solvable Lie group $G$ has
attice.

Let

$$
B_{1}=\left(\begin{array}{ccc}
0 & 0 & 1 \\
1 & 0 & -5 \\
0 & 1 & 6
\end{array}\right), \quad B_{2}=\left(\begin{array}{ccc}
-4 & -4 & -3 \\
21 & 16 & 11 \\
-4 & -3 & -2
\end{array}\right)
$$

Then, $B_{1}, B_{2} \in S L(3, \mathbf{Z})$ satisfy $B_{1} B_{2}=B_{2} B_{1}$, and $\frac{\log \alpha_{1}^{1}}{\log \alpha_{2}^{1}} \neq \frac{\log \alpha_{1}^{2}}{\log \alpha_{2}^{2}}$ (see [5, Prop-
osition 4.4]).

Acknowledgments. The author would like to express his deep appreciation to Professor Yusuke Sakane for valuable advice and encouragement during his 
preparation of the paper. The author also thanks Professor Hiroshi Tamaru and Professor Hiroshi Sawai for several comments and advice.

\section{REFERENCES}

[ 1 ] L. C. de Andrés, L. A. Cordero, M. Fernández and J. J. Mencía, Examples of fourdimensional compact locally conformal Kähler solvmanifolds, Geom. Dedicata 29 (1989), $227-232$.

[2] L. Auslander, An exposition of the structure of solvmanifolds. I. Algebraic theory, Bull. Amer. Math. Soc. 79 (1973), 227-261.

[3] L. Auslander, Lecture notes on nil-theta functions, Regional conference series in mathematics 34, Amer. Math. Soc., Providence, R. I., 1977.

[ 4 ] C. Benson and C. Gordon, Kähler structures on solvmanifolds, Proc. Amer. Math. Soc. 108 (1990), 971-980.

[5] A. Diatta and B. Foreman, Lattices in contact Lie groups and 5-dimensional contact solvmanifolds, Kodai Math. J. 38 (2015), 228-248.

[6] G. D. Mostow, Factor spaces of solvable groups, Ann. of Math. 60 (1954), 1-27.

[7] K. Nomizu, On the cohomology of compact homogeneous spaces of nilpotent Lie groups, Ann. of Math. 59 (1954), 531-538.

[8] M. S. Raghunathan, Discrete subgroups of Lie groups, Ergebnisse der Mathematik und ihrer Grenzgebiete 68, Springer-Verlag, New York-Heidelberg, 1972.

[9] H. SAwaI, A construction of lattices on certain solvable Lie groups, Topology Appl. 154 (2007), 3125-3134.

[10] H. SAwAI, Locally conformal Kähler structures on compact solvmanifolds, Osaka J. Math. 49 (2012), 1087-1102.

[11] H. Sawai and T. Yamada, Lattices on Benson-Gordon type solvable Lie groups, Topology Appl. 149 (2005), 85-95.

Takumi Yamada

Department of Mathematics

Shimane University

NishiKaWATSU-CHO 1060

MATSUE, 690-8504

JAPAN

E-mail: t_yamada@riko.shimane-u.ac.jp 